\title{
Mutual Energy of Relative Motion
}

\author{
Bin-Han Lue ${ }^{1}$, Shyr-Long Jeng ${ }^{2}$ and Wei-Hua Chieng ${ }^{1}$ \\ 1. Department of Mechanical Engineering, National Chiao-Tung University, HsinChu 30010, R.O.C \\ 2. Department of Electrical and Electronic Engineering, Ta Hwa University of Science and Technology, HsinChu 30743. R.O.C
}

\begin{abstract}
A gun, with its explosive energy, can accelerate a bullet, with mass $\mathrm{m}$, from zero velocity. After a bullet is shot into a wall, the wall receives energy, which is converted from explosive to kinetic energy of the ball, and then, to heat and/or deformation energy of the wall. When a person carries this gun in a moving vehicle, and shoots at a wall, the observer witnesses the bullet gain kinetic energy compared with the original scenario. From where is the additional energy derived? Is the explosive energy of the gunpowder a frame-of-reference-dependent quantity? Does it concern the theory of relativity by Albert Einstein? In this article, the additional energy $\mathrm{e}$ is defined as the mutual energy of relative motion, which is a frame-of-reference-dependent energy, and thus, may be transferred from one reference frame to another.
\end{abstract}

Key words: Momentum conservation, energy conservation.

\section{Introduction}

Numerous so-called kinetic energy and reference frame paradoxes [1-6] have been presented on various websites. Certain people doubt that energy is conserved in a moving frame of reference. Others consider the scientific consensus on perpetual motion in an isolated system as a violation of the law of energy conservation [7,8]. Although particular types of perpetual motion have been proven to be impossible [9], numerous others remain mysterious.

Irrespective of the method used for analyzing a mechanical system, when the premises are correct, the equations describing the system should appropriately reflect the conditions. The nature of the mechanical system should not change when we adjust our methods of studying the system. However, on occasion, even for a simple system, the manner of the system motion appears to change depending on the frame of reference chosen to examine the system. When this occurs, it encourages a more thorough study.

Corresponding author: Shyr-Long Jeng, Ph.D., associate professor, research fields: microprocessor based control, and power electronic.

\section{Energy Redistribution Caused by Momentum Conservation}

An isolated vehicle with mass $M$ has an initial velocity $\mathrm{V}$ moving in space when a ball of mass $m$ moves with relative velocity $\mathrm{v}$ in the vehicle, as shown in Fig. 1. In this paper, an isolated system is defined as a dynamic system that is completely enclosed by walls, through which matter, energy, or force cannot pass. The ball then accelerates/decelerates in the vehicle during a period, resulting in a velocity change, $\Delta \mathbf{v}$. The law of momentum conservation is expressed as

$$
m \Delta \mathbf{v}=-M \Delta \mathbf{V}
$$

In Eq. (1), velocity is denoted in vector form to distinguish the direction of velocity. All velocities are collinear and relative to the same frame of reference. The energy required for this relative motion is $\Delta E$, which may be decoupled into three parts:

$$
\begin{aligned}
\Delta E= & \frac{1}{2} m(\mathbf{V}+\mathbf{v}+\Delta \mathbf{v})^{2}-\frac{1}{2} m(\mathbf{V}+\mathbf{v})^{2} \\
& +\frac{1}{2} M(\mathbf{V}+\Delta \mathbf{V})^{2}-\frac{1}{2} M \mathbf{V}^{2} \\
= & \Delta E_{m}+\Delta E_{m, M}+\Delta E_{M}
\end{aligned}
$$




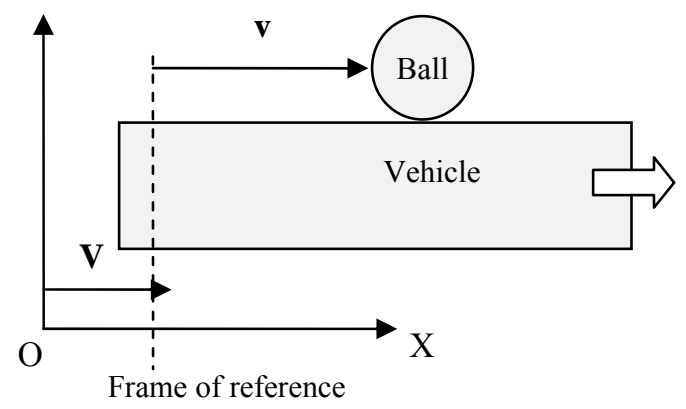

Fig. 1 An isolated system with two masses.

where,

$$
\begin{gathered}
\Delta E_{m}=m(\mathbf{v} \cdot \Delta \mathbf{v})+\frac{1}{2} m(\Delta \mathbf{v})^{2} \\
\Delta E_{m, M}=m(\mathbf{V} \cdot \Delta \mathbf{v}) \\
\Delta E_{M}=M \mathbf{V} \cdot \Delta \mathbf{V}+\frac{1}{2} M(\Delta \mathbf{V})^{2}
\end{gathered}
$$

On the basis of the momentum conservation expressed in Eq. (1), we can obtain

$$
\Delta \mathbf{V}=-\frac{m}{M} \Delta \mathbf{v}
$$

Solving Eq. (3) yields

$$
\Delta E_{M}=-\Delta E_{m, M}+\left(\frac{m}{M}\right) \Delta E_{m, 0}
$$

$\Delta E_{m, 0}$ denotes $\Delta E_{m}$ when $v=0$; that is,

$$
\Delta E_{m, 0}=\frac{1}{2} m(\Delta \mathbf{v})^{2}
$$

$\Delta E_{M}$ may be negative for nonzero $\Delta \mathbf{v}$ when $\mathbf{V} \cdot \Delta \mathbf{v}>0$ and

$$
2 \frac{M}{m}|\mathbf{V}|>|\Delta \mathbf{v}|
$$

where, $\Delta E_{m, M}$ is the energy transferred from the vehicle to the ball, referred to as the mutual energy of relative motion. Although the mutual energies are canceled out within the isolated system according to the energy equation of Eq. (2), the vehicle provides the ball with this mutual energy with respect to the reference frame. The mutual energy of relative motion is an internal energy within an isolated system, and it is induced by energy redistribution resulting from changes in the momentum of different masses within the isolated system. In actuality, the vehicle decelerates when the ball accelerates, and the ball decelerates when the vehicle accelerates. Mutual energy depends on absolute velocity $\mathbf{V}$. Because velocity is defined according to the reference frame, it is a frame-of-reference -dependent quantity.

\section{Energy Efficiency}

The total kinetic energy gained by the ball is

$$
\begin{aligned}
W_{K E} & =\Delta E_{m}+\Delta E_{m, M} \\
& =\Delta E_{m, 0}+m(\mathbf{v} \cdot \Delta \mathbf{v})+m(\mathbf{V} \cdot \Delta \mathbf{v})
\end{aligned}
$$

The effort expended to accelerate/decelerate the ball, which may be converted from some form of potential energy, is expressed as

$$
\begin{aligned}
W_{P E} & =\Delta E=\Delta E_{m}+\Delta E_{m, M}+\Delta E_{M} \\
& =\left(1+\frac{m}{M}\right) \Delta E_{m, 0}+m(\mathbf{v} \cdot \Delta \mathbf{v})
\end{aligned}
$$

The energy efficiency in converting the input energy into incremental kinetic energy is derived as follows:

$$
\eta=\frac{W_{K E}}{W_{P E}}=\frac{\Delta E_{m, 0}+m(\mathbf{v} \cdot \Delta \mathbf{v})+m(\mathbf{V} \cdot \Delta \mathbf{v})}{\left(1+\frac{m}{M}\right) \Delta E_{m, 0}+m(\mathbf{v} \cdot \Delta \mathbf{v})}
$$

In the particular case that $\mathbf{v}=0$, efficiency may be derived as

$$
\eta=\frac{1}{(1+m / M)} \pm \frac{2|\mathbf{V}|}{|\Delta \mathbf{v}|}
$$

The plus/minus sign in this equations depends on both the direction of and the change in velocity. When the ball is thrown in the same direction of the moving vehicle, during the actual throwing motion, it is highly efficient. As another example, if a person can run sufficiently fast in a direction to cause the Earth to stop spinning, then the Earth provides that person with all of its kinetic energy. This result is expected because numerous perpetual motion apparatuses are derived from this phenomenon, in which kinetic energy is acquired from the Earth.

Another question is as follows: Can the ball hold 
mutual energy as long as it remains on the vehicle? The answer is no. In other words, when the ball is coming to rest relative to the vehicle, it returns the mutual energy to the vehicle because of the sign change of $\mathbf{V} \cdot \Delta \mathbf{v}$.

Example 1. In Ref. [6], as shown in Fig. 2, the statement is simplified to the following: "Because kinetic energy $=\frac{1}{2} m v^{2}, 1 \mathrm{~J}$ is required to accelerate a 2-kg ball from 0 to $1 \mathrm{~m} / \mathrm{s}$. For example, a passing train with velocity $1 \mathrm{~m} / \mathrm{s}$ is moving in the same direction as the mass. Let a person on the moving train throw a ball with an acceleration of $2 \mathrm{~m} / \mathrm{s}$. Only one additional joule of energy is required to accelerate the ball from 0 to $1 \mathrm{~m} / \mathrm{s}$ on the train, but it requires three additional joules for people on the ground to accelerate the mass from 1 to $2 \mathrm{~m} / \mathrm{s}$, as implied by the kinetic energy equation-but why? First, the relative velocity between the ball and the train is zero when the person begins holding the ball. Therefore, $\mathbf{v}=0$. The answer can be derived from Eq. (6); the total kinetic energy of the ball is identical to that of both people on the ground and the person on the train and can thus be expressed as $\quad W_{K E}=\frac{1}{2} m(\Delta \mathbf{v})^{2}+m(\mathbf{V} \cdot \Delta \mathbf{v})=\frac{1}{2} 2(1)^{2}+2(1 \cdot 1)=3 \quad \mathrm{~J}$.

The train contributes $2 \mathrm{~J}$ of mutual energy of relative motion to the kinetic energy of the ball, according to Eqs. (3) and (4). The person on the train must provide $W_{P E}=\left(1+\frac{2}{M}\right) \frac{1}{2} 2(1)^{2}=\left(1+\frac{2}{M}\right) \quad$ joules, instead of $1 \mathrm{~J}$, according to Eq. (7). Among the total input energy $W_{P E}, \frac{2}{M} \mathrm{~J}$ is provided to the train. The overall energy efficiency of the people on the ground is identical when the person on the train is $\eta=2+\frac{1}{(1+m / M)}$, according to Eq. (8), which is nearly triple the efficiency of the people on the ground.

Example 2. Is the gun powder energy a frame-of -reference-dependent quantity? As shown in Fig. 3,

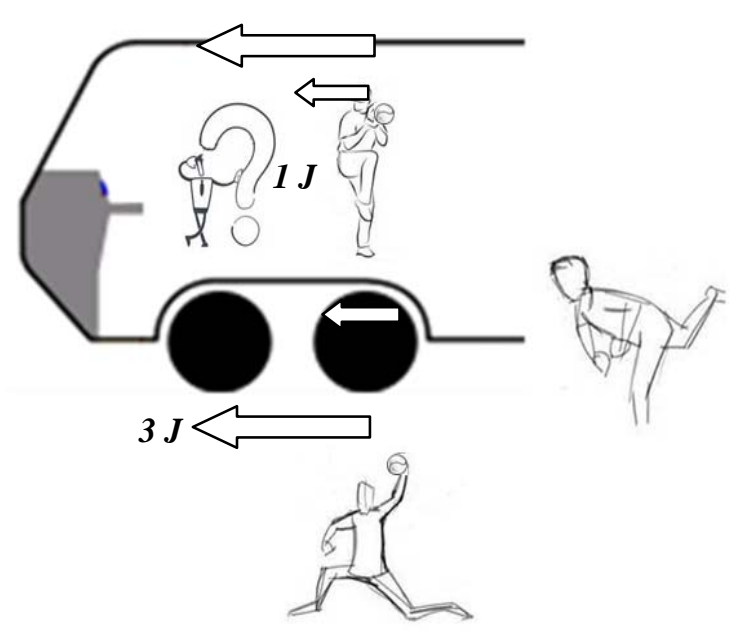

Fig. 2 Equal final velocity by different energy inputs on different reference frames.

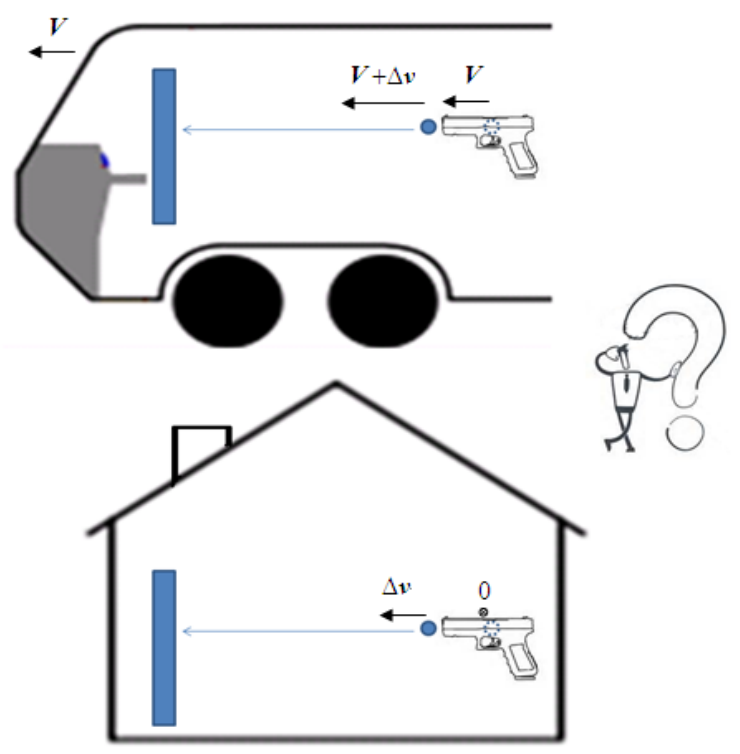

Fig. 3 Same gun shooting in different reference frames.

assuming that the gun powder has explosive energy $E_{G}$ when the gun is onboard a train, the kinetic energy of the bullet increases by $\eta E_{G}$ when the gun is fired on the train, according to Eq. (8). According to Eq. (6), when the bullet travels in the same direction as the train,

$$
\eta E_{G}=W_{K E}=\Delta E_{m, 0}+m(\mathbf{V} \cdot \Delta \mathbf{v})
$$

According to Eq. (7),

$$
E_{G}=W_{P E}=\left(1+\frac{m}{M}\right) \Delta E_{m, 0}
$$

The energy of the gun powder was found not to be a 
frame-of-reference-dependent quantity because the energy converted from $E_{G}$ into kinetic energy was independent of the train velocity $\mathbf{V}$. On the basis of the assumption that the mass ratio between the bullet and the train was negligible, we obtained $E_{G} \approx \Delta E_{m, 0}$. The explosive energy of the gun powder $E_{G}$ contributed an incremental amount of kinetic energy of the bullet independently from the frames of reference.

The loss of kinetic energy from the bullet decelerating during the collision and finally stopping when it hits the wall may be derived from Eq. (6):

$$
W_{K E}=\frac{1}{2} m\left(\Delta \mathbf{v}_{2}\right)^{2}+m\left(\mathbf{v} \cdot \Delta \mathbf{v}_{2}\right)+m\left(\mathbf{V}_{2} \cdot \Delta \mathbf{v}_{2}\right)
$$

In this equation, $\mathbf{v}$ denotes the velocity of the bullet after the gun is fired, $\Delta \mathbf{v}_{2}$ represents the amount of velocity reduction when the bullet hits the wall, and $\mathbf{V}_{2}$ denotes the velocity of the vehicle after the gun is fired. The bullet accelerated to a velocity $\mathbf{v}=\Delta \mathbf{v}$ relative to the train after the gun was fired. For a small mass ratio, the train velocity merely changed before and after the gun was fired (i.e., $\mathbf{V}_{2} \approx \mathbf{V}$ and $\left.\Delta \mathbf{v}_{2} \approx-\Delta \mathbf{v}\right)$. The change in kinetic energy expressed in Eq. (10) may be rewritten as follows:

$$
\begin{aligned}
W_{K E} & =-\Delta E_{m, 0}+m(\mathbf{V} \cdot \Delta \mathbf{v}) \\
& \approx-\Delta E_{m, 0}-m(\mathbf{V} \cdot \Delta \mathbf{v})=-\eta E_{G}
\end{aligned}
$$

This equation shows that the mutual energy of relative motion in the bullet returned immediately to the train from the free space. The next question may concern whether the wall incurs greater damage when the gun is fired from inside the train, which can be addressed using Eq. (7).

$$
\begin{aligned}
W_{P E} & =\left(1+\frac{m}{M}\right) \Delta E_{m, 0}-m\left(\mathbf{v}_{2} \cdot \Delta \mathbf{v}_{2}\right) \\
& =\left(-1+\frac{m}{M}\right) \Delta E_{m, 0} \approx-\Delta E_{m, 0}=-E_{G}
\end{aligned} .
$$

This equation displays that the total heat and/or deformation energy of the wall are derived from the explosive energy of the gun powder $E_{G}$. This is identical to the result of when the gun was fired from the ground. The damage to two walls on either the ground or the train is similar.

\section{Application}

As discussed in the previous sections, mutual energy is a frame-of-reference-dependent quantity. Once an object has a velocity relative to the reference frame, mutual energy is imposed onto the object. As mentioned in Section 2, mutual energy is returned to the reference frame when the object becomes still. Although numerous types of perpetual motion are based on mutual energy for creating the illusion of endless energy sources, it does not weaken the use of mutual energy. The object acquires mutual energy from the reference frame, and it must eventually return to the frame. If a price difference exists between buying and selling energy, then securing concerted advantages from the mutual energy may be possible.

Example 3. As shown in Fig. 4, a rotating machine of mass $\mathrm{M}$ has multiple containers. Within each container is a ball with mass $\mathrm{m}$, and $\mathrm{m}$ is substantially smaller than $\mathrm{M}$. The ball is ejected with velocity $\Delta v$ relative to the container when it receives energy. Assuming that the machine rotates with an angular velocity $\Omega$, each container has a linear velocity $\mathrm{V}$. The generator catches the ball and converts the kinetic energy $\frac{1}{2} m(\Delta v)^{2}+m V \Delta v$ into electrical energy. To return the machine to its original state, the generator becomes a motor that converts electrical energy back to the kinetic energy required for returning each ball, with velocity $\mathrm{V}+\Delta v$, to the individual containers. The amount of mutual energy can be massive when $\mathrm{V}>>\Delta v$.

The price of electricity is known to be a function of time. The price is higher during the day than at night. It is preferable to return the balls to the machine at night to incur a lower electricity fee and to generate electricity during the day. The difference in electricity prices grants the operator the operating advantage. 


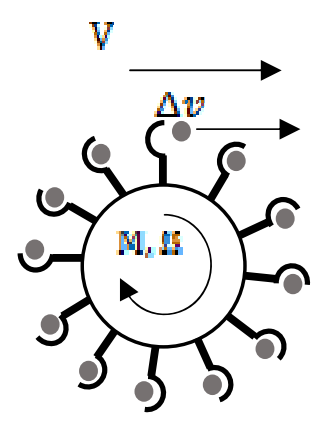

Generator

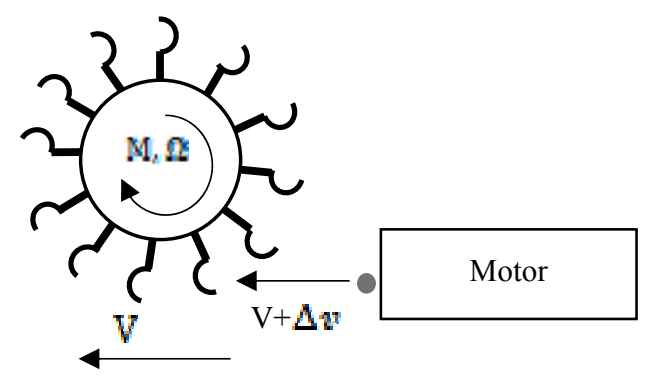

Fig. 4 Generator/motor perpetual motion.

\section{Conclusion}

The mutual energy of relative motion is an internal energy within an isolated system and depends on the frame of reference, which does not concern the theory of relativity by Albert Einstein. The mutual energy of relative motion may be conveyed from a vehicle to the reference frame by using procedures analogous to Example 1. Numerous perpetual motion apparatuses have been derived to acquire mutual energy from the Earth. These do not involve true perpetual motion and instead perturb the Earth's motion. Obtaining mutual energy from moving vehicles during the day, when electricity prices are higher than at night, may be useful. We hope that innovative machines or mechanisms with microwave energy transfer can be invented to efficiently use this mutual energy of relative motion.

\section{Acknowledgment}

This work was supported by the Ministry of Science and Technology project MOST 104-2221-E-009-122-, Taiwan, R.O.C. The authors thank Kuo-Tung Tsai for the helpful suggestions and technical support.

\section{References}

[1] http://physics.stackexchange.com/questions/51220/kineti c-energy-with-respect-to-different-reference-frames, January, 2013.

[2] http://physics.stackexchange.com/questions/23734/is-theenergy-conserved-in-a-moving-frame-of-reference, February, 2015.

[3] https://www.physicsforums.com/threads/mechanical-ener gy-and-frames-of-reference.250374/, February, 2015.

[4] https://www.physicsforums.com/threads/kinetic-energy-h eat-and-reference-frame.417355/, February, 2015.

[5] http://newrelativity.net/yahoo_site_admin/assets/docs/Mo mentumParadoxNotes.146112626.pdf, February, 2015.

[6] https://www.physicsforums.com/threads/kinetic-energy-a nd-frames-of-reference.534883/, February, 2015.

[7] Stanley, A. 1968. "Perpetual Motion Machines." Scientific American 218 (1): 115-22.

[8] James, K. 2008. "An Energy Storage Device and Method of Use.” WO2008037004 (A1), 2008-04-03.

[9] Jenkins, A. 2013. "Self-oscillation." Physics Reports 525 (2): 167-222. 